\title{
Two-dimensional fractal analysis of retinal tissue of healthy and diabetic eyes with optical coherence tomography
}

\author{
Wei Gao ${ }^{1 *}$, Delia Cabrera DeBuc ${ }^{2}$, Valery P. Zakharov ${ }^{3}$, Erika Tatrai ${ }^{4}$, Gabor M. Somfai ${ }^{4}$, \\ Oleg O. Myakinin ${ }^{3}$, Ivan A. Bratchenko ${ }^{3}$, Dmitry N. Artemyev ${ }^{3}$, and Dmitry V. Kornilin ${ }^{3}$ \\ ${ }^{1}$ School of Safety Engineering, Ningbo University of Technology, China \\ 2 Bascom Palmer Eye Institute, University of Miami, Florida, United States of America \\ ${ }^{3}$ Department of Laser and Biotechnical Systems, Samara National Research University, Russian Federation \\ ${ }^{4}$ Department of Ophthalmology, Semmelweis University, Budapest, Hungary \\ *e-mail: gwei@ustc.edu
}

\begin{abstract}
In the ophthalmic research, the measurement of the retinal thickness is usually employed for characterizing the structural changes of the retinal tissue. However, changes in the fractal dimension (FD) may provide additional information regarding the structure of the retinal layers and their early damage in ocular diseases. In the present paper, we investigated the possibility of detecting changes in the structure of the cellular layers of the retina by applying a two-dimensional fractal analysis to optical coherence tomography (OCT) images. OCT images were obtained from diabetic patients without retinopathy (DM, $n=38$ eyes) and with mild diabetic retinopathy (MDR, $n=43$ eyes) as well as in healthy subjects (Controls, $n=74$ eyes). The two-dimensional fractal dimension was calculated using the differentiate box counting methodology. We evaluated the usefulness of quantifying the fractal dimension of layered structures in the detection of retinal damage. Generalized estimating equations considering within-subject inter-eye relations were used to test for differences between the groups. An adjusted $p$-value of $<0.001$ was considered statistically significant. Receiver operating characteristic (ROC) curves were constructed to describe the ability of the fractal dimension to discriminate between the eyes of DM, MDR, and healthy eyes. Lower values of the fractal dimension were observed in all layers in the MDR eyes compared with controls except in the inner nuclear layer (INL). Lower values of the fractal dimension were also found in all layers in the MDR eyes compared with DM eyes. The highest area under receiver operating characteristic curve (AUROC) values estimated for the fractal dimension were observed for the outer plexiform layer (OPL) and outer segment photoreceptors (OS) when comparing MDR eyes with controls. The highest AUROC value estimated for the fractal dimension were also observed for the retinal nerve fiber layer (RNFL) and OS when comparing MDR eyes with DM eyes. Our results suggest that fractal dimension of the intraretinal layers may provide useful information to differentiate pathological from healthy eyes. Further research is warranted to determine how this approach may be used to aid diagnosis of retinal neurodegeneration at the early stage. (C) 2016 Journal of Biomedical Photonics \& Engineering.
\end{abstract}

Keywords: optical coherence tomography; retina; diabetic retinopathy; fractal analysis, differentiate box counting.

Paper \#3114 received 2016.11.09; accepted for publication 2016.12.30; published online 2016.12.31. doi: $10.18287 / J B P E 16.02 .040302$ 


\section{References}

1. D. Huang, E. A. Swanson, C. P. Lin, J. S. Schuman, W. G. Stinson, W. Chang, M. R. Hee, T. Flotte, K. Gregory, C. A. Puliafito, and J. G. Fujimoto, “Optical coherence tomography," Science 254(5035), 1178-1181 (1991).

2. D. C. DeBuc, and G. M. Somfai, "Early detection of retinal thickness changes in diabetes using optical coherence tomography," Medical Science Monitor 16(3), Mt15-21 (2010).

3. E. Tatrai, M. Simo, A. IIjicsov, J. Nemeth, D. C. DeBuc, and G. M. Somfai, "In vivo evaluation of retinal neurodegeneration in patients with multiple sclerosis," PLoS One 7(1) e30922 (2012).

4. D. C. DeBuc, "Novel methods and diagnostic tools in diabetic retinopathy," Retinal Physician 12, 22-27 (2015).

5. G. M. Somfai, E. Tatrai, L. Laurik, B. E. Varga, V. Olvedy, H. Jiang, J. H. Wang, W. E. Smiddy, A. Somogyi, and D. C. DeBuc, "Automated classifiers for early detection and diagnosis of retinopathy in diabetic eyes," BMC Bioinformatics 15(1), 106 (2014).

6. G. M. Somfai, E. Tatrai, L. Laurik, B. E. Varga, V. Olvedy, W. E. Smiddy, R. Tchitnga, A. Somogyi, and D. C. DeBuc, "Fractal-based analysis of optical coherence tomography data to quantify retinal tissue damage," BMC Bioinformatics 15, 295 (2014).

7. D. C. DeBuc, E. Tatrai, L. Laurik, B. E. Varga, V. Olvedy, A. Somogyi, W. E. Smiddy, and G. M. Somfai, "Identifying local structural and optical derangement in the neural retina of individuals with type 1 diabetes," Journal of Clinical and Experimental Ophthalmology 4(4) 1000289 (2013).

8. U. H. Schaudig, C. Glaefke, F. Scholz, and G. Richard, "Optical coherence tomography for retinal thickness measurement in diabetic patients without clinically significant macular edema," Ophthalmic Surgery Lasers 31(3), 182-186 (2000).

9. T. Oshitari, K. Hanawa, and E. Adachi-Usami, "Changes of macular and RNFL thicknesses measured by Stratus OCT in patients with early stage diabetes," Eye (London) 23(4), 884-889 (2009).

10. B. Asefzadeh, B. M. Fisch, C. E. Parenteau, and A. A. Cavallerano, "Macular thickness and systemic markers for diabetes in individuals with no or mild diabetic retinopathy," Clinical \& Experimental Ophthalmology 36(5), 455-463 (2008).

11. W. Goebel, and T. Kretzchmar-Gross, "Retinal thickness in diabetic retinopathy: a study using optical coherence tomography (OCT)," Retina 22(6), 759-767 (2002).

12. N. M. Bressler, A. R. Edwards, A. N. Antoszyk, R. W. Beck, D. J. Browning, A. P. Ciardella, R. P. Danis, M. J. Elman, S. M. Friedman, A. R. Glassman, J. G. Gross, H. K. Li, T. J. Murtha, T. W. Stone, and J. K. Sun, "Retinal thickness on Stratus optical coherence tomography in people with diabetes and minimal or no diabetic retinopathy," American Journal of Ophthalmology 145(5), 894-901 (2008).

13. P. G. H. Clarke, "Developmental cell death: morphological diversity and multiple mechanisms," Anatomy and Embryology 181(3) 195-213 (1990).

14. C. D. M. Fletcher (ed.), Diagnostic histopathology of tumors, 2nd. ed., Churchill Livingstone, London (2000). ISBN: 978-0-443-07992-4.

15. G. M. Somfai, H. M. Salinas, C. A. Puliafito, and D. C. Fernandez, "Evaluation of potential image acquisition pitfalls during optical coherence tomography and their influence on retinal image segmentation," Journal of Biomedical Optics 12(4), 041209 (2007).

16. D. C. DeBuc, System and method for early detection of diabetic retinopathy using optical coherence tomography. U.S. Patent WO2010080576 (2010).

17. D. C. DeBuc, H. M. Salinas, and C. A. Puliafito, "Automated detection of retinal layer structures on optical coherence tomography images,” Optics Express 13(25), 10200-10216 (2005).

18. C. Flueraru, D. P. Popescu, Y. Mao, S. Chang, and M. G. Sowa, "Added soft tissue contrast using signal attenuation and the fractal dimension for optical coherence tomography images of porcine arterial tissue," Physics in Medicine and Biology 55(8), 2317-2331 (2010).

19. A. C. Sullivan, J. P. Hunt, and A. L. Oldenburg, "Fractal analysis for classification of breast carcinoma in optical coherence tomography," Journal of Biomedical Optics 16(6), 066010 (2011).

20. W. Gao, Improving the quantitative assessment of intraretinal features by determining both structural and optical properties of the retinal tissue with optical coherence tomography, Ph.D thesis, University of Miami (2012).

21. M. Hasegawa, J. Liu, K. Okuda, and M. Nunobiki, "Calculation of the fractal dimensions of machined surface profiles," Wear 192(1-2), 40-45 (1996).

22. N. Sarkar, and B. B. Chaudhuri, "An efficient approach to estimate fractal dimension of textural images," Pattern Recognition 25(9), 1035-1041 (1992).

23. J. Li, Q. Du, and C. Sun, “An improved box-counting method for image fractal dimension estimation,” Pattern Recognition 42(11), 2460-2469 (2009). 
24. A. J. Barber, E., Lieth, S. A. Khin, D. A. Antonetti, A. G. Buchanan, and T. W. Gardner, "Neural apoptosis in the retina during experimental and human diabetes. Early onset and effect of insulin," The Journal of Clinical Investigation 102 (4), 783-791 (1998).

25. S. H. Park, J. W. Park, S. J. Park, K. Y. Kim, J. W. Chung, M. H. Chun, and S. J. Oh, “Apoptotic death of photoreceptors in the streptozotocin-induced diabetic rat retina," Diabetologia 46(9), 1260-1268 (2003).

26. E. Rungger-Brandle, A. A. Dosso, and P. M. Leuenberger, "Glial reactivity, an early feature of diabetic retinopathy," Investigative Ophthalmology and Visual Science 41(7), 1971-1980 (2000).

27. X. X. Zeng, Y. K. Ng, and E. A. Ling, "Neuronal and microglial response in the retina of streptozotocininduced diabetic rats," Visual Neuroscience 17(3), 463-471 (2000).

28. A. J. Barber, D. A. Antonetti, T. S. Kern, C. E. N. Reiter, R. S. Soans, J. K. Krady, S. W. Levison, T. W. Gardner, and S. K. Bronson, "The Ins2 $2^{\text {Akita }}$ mouse as a model of early retinal complications in diabetes," Investigative Ophthalmology and Visual Science 46(6), 2210-2218 (2005).

29. B. J. Lujan, A. Roorda, J. A. Croskrey, A. M. Dubis, R. F. Cooper, J. K. Bayabo, J. L. Duncan, B. J. Antony, J. Caroll, "Directional optical coherence tomography provides accurate outer nuclear layer and Henle fiber layer measurements," Retina 35(8), 1511-1520 (2015).

30. T. Otani, Y. Yamaguchi, and S. Kishi, "Improved visualization of Henle fiber layer by changing the measurement beam angle on optical coherence tomography," Retina 31(3), 497-501 (2011).

31. R. Akshikar, M. Richardson, R. Crosby-Nwaobi, R., A. Abdelhay, S. Sivaprasad, S. Heng, "Retinal neuronal changes in people with diabetes," Investigative Ophthalmology and Visual Science 53, 2852 (2012).

32. A. Verma, P. K. Rani, R. Raman, S. S. Pal, G. Laxmi, M. Gupta, C. Sahu, K. Vaitheeswaran, and T. Sharma, "Is neuronal dysfunction an early sign of diabetic retinopathy? Microperimetry and spectral domain optical coherence tomography (SD-OCT) study in individuals with diabetes, but no diabetic retinopathy," Eye (London) 23(9), 1824-1830 (2009).

33. P. M. Martin, P. Roon, T. K. Van Ells, V. Ganapathy, and S. B. Smith, "Death of retinal neurons in streptozotocin-induced diabetic mice," Investigative Ophthalmology and Visual Science 45(9), 3330-3336 (2004).

34. G. Liew, J. J. Wang, P. Mitchell, T. Y. Wong, "Retinal vascular imaging: a new tool in microvascular disease research," Circulation Cardiovascular imaging 1(2), 156-161 (2008).

35. M. Hasegawa, J. Liu, K. Okuda, M. Nunobiki, "Calculation of the fractal dimensions of machined surface profiles,"Wear 192(1), 40-45 (1996).

\section{Introduction}

Optical coherence tomography is an optical imaging modality that provides high-resolution, cross-sectional images of biological tissue in a non-invasive manner [1]. Notably, the OCT technology has been widely utilized to detect retinal diseases in Ophthalmology. By employing the OCT technique, the thickness and volume measurements of the retinal tissue can be obtained from OCT scans. Particularly, the structural alterations revealed by changes in thickness and volume of the cellular layers of the retina can be measured to characterize the neurodegeneration in patients with diabetes [2-7]. The most significant retinal pathology caused by diabetes is diabetic retinopathy (DR), which is characterized by blood vessel damage and neurodegenerative changes.

The quantification of the thickness measurement of various cellular layers of the retina with OCT has helped to assess treatment efficacy and identify potential markers for monitoring the progression of DR [8-12]. However, in biology and medicine, the shapes of structures such as molecules, cells, tissues, and organs also play a significant role in the diagnosis of diseased retinal tissue [13-14]. Out of the various structural parameters, the fractal dimension can be capable of revealing differences and irregularities in these structures. Quantitative measurements of the fractal dimension could be an effective approach to discriminate diseased tissue from healthy tissue. Therefore, the structural changes revealed by changes in fractal dimension may provide further information regarding cellular layers and early damage in ocular diseases. The abnormal retinal tissue could be detected by performing fractal analysis for particular cellular structures visualized in OCT images. Thus, fractal analysis of OCT images could provide a useful diagnostic methodology to identify diseased tissue.

In this study, OCT images were obtained from diabetic and normal healthy subjects to investigate the possibility of OCT to detect structural changes in the retina by quantifying the two-dimensional fractal dimension of its layered structures.

\section{Materials and Methodology}

\subsection{Data Collection}

The data collection included participants recruited under a study sponsored by the Juvenile Diabetes Research Foundation (JDRF). The Institutional Review Board of each institution (Bascom Palmer Eye Institute at University of Miami, FL, USA and Semmelweis University, Budapest, Hungary) involved in the study approved the study protocol. The research adhered to the tenets outlined in the Declaration of Helsinki. Informed consent was obtained from each subject. OCT examination was performed in healthy and diabetic eyes 
with and without retinopathy. Once the subject was enrolled in the study, one visit was required to perform a comprehensive eye examination including the following assessments: best-corrected visual acuity, intraocular pressure (using a Goldmann tonometer) and seven standard field stereoscopic fundus photos. Also, hemoglobin A1c test was required at this visit for diabetic patients with no past glycemic control. No additional tests were needed after this first visit or during the time the study was completed. Inclusion

Table 1 Characteristics of the study participants.

\begin{tabular}{cccc}
\hline Characteristic & Controls & DM & MDR \\
\hline Number of Participants & 41 & 29 & 29 \\
Number of Eyes & 74 & 38 & 43 \\
Age (years, mean \pm SD) & $34 \pm 12$ & $35 \pm 10$ & $43 \pm 17$ \\
Female, N (\% total eyes) & $52(70 \%)$ & $20(53 \%)$ & $21(49 \%)$ \\
Race (\% Caucasian) & 100 & 100 & 91 \\
Hemoglobin Alc level (\%) & - & $7.20 \pm 0.90$ & $8.51 \pm 1.76$ \\
DM duration (years, mean \pm SD) & - & $13 \pm 5$ & $22 \pm 10$ \\
IOP (mmHg, mean \pm SD) & - & $15.74 \pm 1.77$ & $15.09 \pm 1.56$ \\
BCVA & $1.00 \pm 0.00$ & $1.00 \pm 0.00$ & $0.97 \pm 0.06$ \\
\hline
\end{tabular}

Abbreviations: SD: standard deviation; DM: diabetic eyes without retinopathy; MDR: diabetic eye with mild diabetic retinopathy; BCVA: best corrected visual acuity

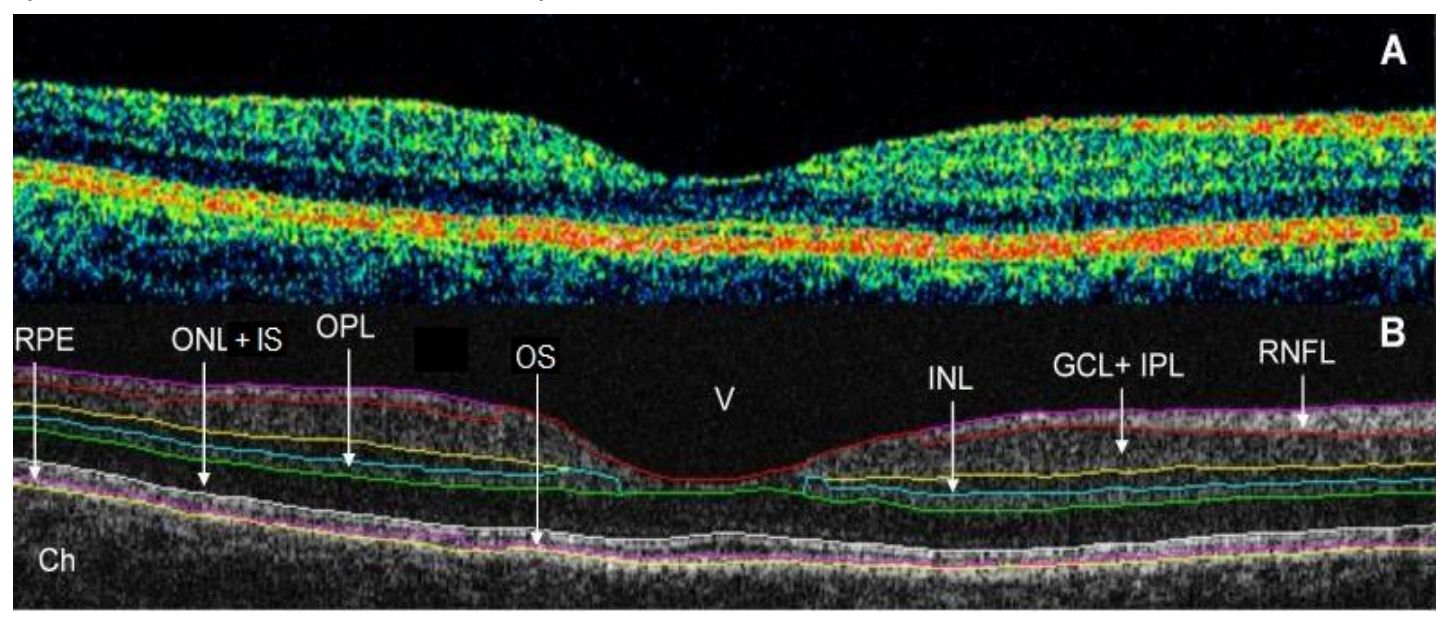

Fig. 1 Macular image segmentation using OCTRIMA. (A) The image of a healthy macula scanned by Stratus OCT. (B) The same OCT scan processed with OCTRIMA. Abbreviations: Ch, choroid; GCL+IPL, ganglion cell layer and inner plexiform layer complex; INL, inner nuclear layer; ONL+IS, combined outer nuclear layer and inner segment of photoreceptors; OS, outer segment of photoreceptors; OPL, outer plexiform layer; RNFL, retinal nerve fiber layer; RPE, retinal pigment epithelial layer; V, vitreous. Note that OCTRIMA measures the thickness of the whole retina between the inner limiting membrane and the inner boundary of the photoreceptor outer segment/RPE junction. The thickness of the combined ONL+IS structure is measured between the outer boundary of OPL and the inner boundary of the photoreceptor outer segment/RPE junction.

criteria for healthy controls included best-corrected visual acuity of $20 / 25$ or better, no history of any current ocular or systematic disease and a normalappearing macula on contact lens biomicroscopy. All eye examinations were performed at the Department of Ophthalmology, Semmelweis University, Budapest, Hungary. A total of 74 normal healthy eyes, 38 eyes with type 1 diabetes mellitus with no retinopathy and 43 eyes with mild diabetic retinopathy on biomicroscopy were included in this study (see Table 1).

\subsection{OCT System and Measurements}

The OCT system (Stratus OCT, Carl Zeiss Meditec, Dublin, California) used in this study employs a broadband light source, delivering an output power of $1 \mathrm{~mW}$ at the central wavelength of $820 \mathrm{~nm}$ with a bandwidth of $25 \mathrm{~nm}$. It has a scanning speed of 400 A-scans per second with a resolution of $10 \mu \mathrm{m}$ in tissue that determines the imaging axial resolution of the system. A cross-sectional image is achieved by the axial reflectance while the sample is scanned laterally. All Stratus OCT study cases were obtained using the macular thickness map protocol. This protocol consists of six radial scan lines centered on the fovea, each having a $6 \mathrm{~mm}$ transverse length. To obtain the best image quality, focusing and optimization settings were controlled, and scans were accepted only if the signal strength was above 6 (preferably 9-10) [15]. Scans with 
foveal decentration (i.e., with center point thickness $\mathrm{SD}>10 \%$ ) were repeated.

\subsection{OCT image processing}

OCT signals were collected and exported from the OCT device in the form of 16-bit grayscale images. To use OCT to quantitatively evaluate structural changes of the cellular layers of the retina caused by some severe eye diseases, a segmentation of these layers is required. We used a computer-aided grading methodology for OCT retinal image analysis (OCTRIMA) that is an interactive, user-friendly stand-alone application for analyzing Stratus OCT retinal images [16]. This method integrates a denoising and edge enhancement technique along with a segmentation algorithm previously developed by Cabrera et al [17]. The denoising and edge enhancement techniques are part of a novel preprocessing step that facilitates better automatic segmentation results (see Figure 1). Also, the semiautomatic segmentation correction tool minimizes segmentation errors generated during the automatic segmentation process, significantly reducing the need for manual error corrections. It also gives quantitative information about intraretinal structures and facilitates the analysis of other retinal features that may be of diagnostic and prognostic value, such as morphology and reflectivity.

\subsection{Fractal Analysis}

In the analysis of OCT images, fractal analysis has been used to investigate the structural change of biological tissue. For example, Fluearu et al. used the box counting method to calculate the fractal dimension to characterize porcine arterial tissue [18]. Sullivan et al. utilized the box counting method to calculate the fractal dimension to classify the breast carcinoma [19]. The power spectrum method has been used to perform the fractal analysis on the layered retinal tissue for investigating the diseased tissue in diabetic patients and healthy subjects [5-7,17,20]. In those studies, the fractal analysis was performed on each A-scan within each region of interest (ROI). Therefore, only the irregularity or roughness along the direction of A-scans was considered in the one-dimensional fractal analysis, while the irregularity or unevenness in all other directions was ignored. Therefore, to extend the fractal analysis in all directions in OCT images and to improve the accuracy of the fractal analysis, the two-dimensional fractal analysis for intraretinal layers of OCT images is necessary.

To determine the fractal dimension of 2D OCT images, several definitions of fractal dimension have been used [21]. One straightforward and understandable methodology for calculating the $2 \mathrm{D}$ fractal dimension is the differentiate box counting method [22]. The method of differentiate box counting method is described below.

Consider a grayscale image in a Cartesian coordinate $(\mathrm{x}, \mathrm{y}, \mathrm{z})$, the coordinates $(\mathrm{x}, \mathrm{y})$ denote the $2 \mathrm{D}$ position on the image plane, and the coordinate $(\mathrm{z})$ represents the intensity value. In the $2 \mathrm{D}$ differentiate box-counting method, the image plane is covered by the nonoverlapping grids. Assume the grayscale image's size is $W \times W$ and the non-overlapping grid's size is $s \times s$, $W$ and $s$ need to meet the requirement as $W / 2 \geq s \geq 2$. Assume the $G$ is the total number of gray level, the value $s^{\prime}$ can be derived from the following equation: $s^{\prime}=G s / W$. Thus, the size of the box that is used to cover the 3D spatial surface is $s \times s \times s^{\prime}$ as showed in Figure 2. Here, assume that the minimum and maximum gray level in the $(i, j)$ grid were in the box number $k$ and $l$, respectively, the number of the boxes covering the surface in the grid $(i, j)$ is:

$$
n_{r}(i, j)=l-k+1 \text {. }
$$

Taking contributions across all grids, we have

$$
N_{r}=\sum_{i, j} n_{r}(i, j)
$$

where $N_{r}$ is counted for different values of $r$. Then, the fractal dimension of 2D OCT image can be estimated from the least square linear fit of $\log \left(N_{r}\right)$ versus $\log (1 / r)$.

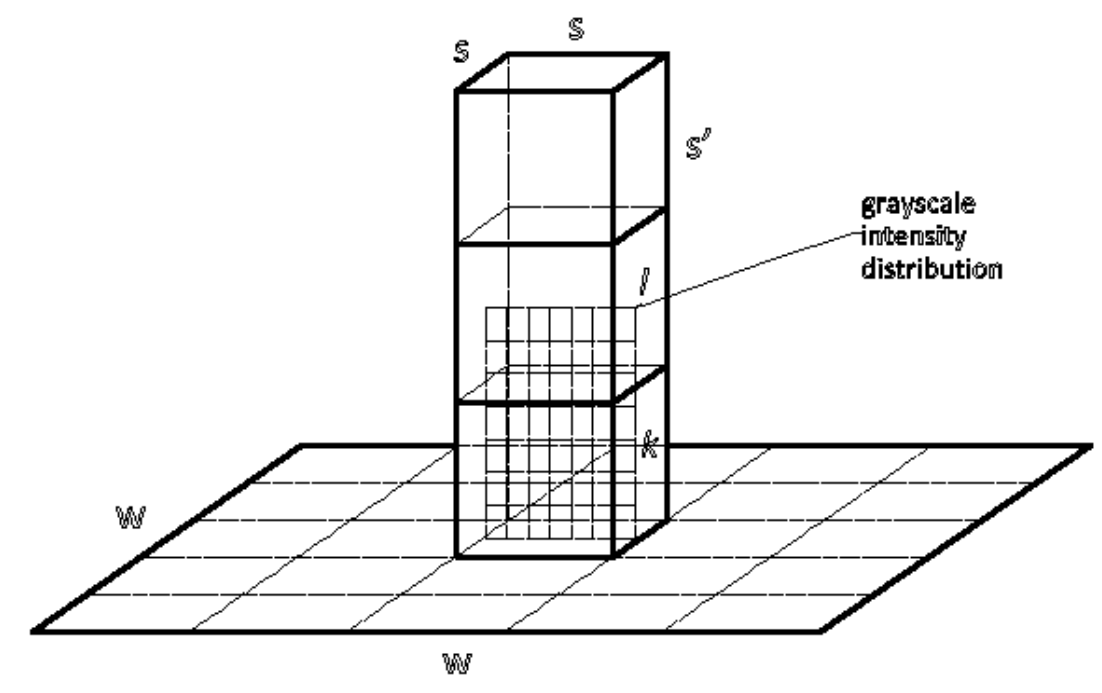

Fig. 2 Sketch of determination of the number of boxes by the differentiate box counting method [23]. 


\subsection{Statistical Analysis}

The differences in fractal dimension between study groups were tested using an ANOVA followed by Newman-Keuls posthoc analysis and the student's t-test. Because of the number of comparisons, $p \leq 0.001$ was considered statistically significant. Receiver operating characteristic analysis was also performed on the fractal dimension between study groups to determine the ability of fractal dimension to differentiate diabetic eyes with and without MDR from healthy eyes. AUROC was used to compare diagnostic power. This area summarizes the sensitivity and specificity of diagnosis over the total range of valid diagnostic thresholds. An AUROC of 1.0 indicated perfect discrimination. An AUROC of 0.5 indicated no discrimination. The AUROC calculations and statistical analyses were performed using the software package SPSS version 16 (SPSS Inc., Chicago, Illinois).

\section{Results and Discussion}

OCT is usually employed for the measurement of retinal thickness. However, the fractal analysis may provide further information regarding cellular layers and early damage in ocular diseases. In this study, the fractal dimension showed the most powerful diagnostic utility for detecting early changes in the diabetic retina.

Significantly smaller values of fractal dimension were observed in all layers in the MDR eyes compared with controls (except INL, see Table 2). Specifically, the fractal dimension was $2.0 \%$ smaller in the OPL as compared to healthy eyes. When comparing MDR with DM eyes, the fractal dimension had a significantly less value in all intraretinal layers. Specifically, the fractal dimension was $1.7 \%$ smaller in the RNFL. Our results suggest that the RNFL, OPL, ONL+IS, and OS are more susceptible to initial damage when comparing MDR with control eyes. Mainly, the trend observed for the fractal dimension of the RNFL in MDR eyes might be associated with pathological metabolic changes in the retina and may reflect neurodegenerative changes in the diabetic retina. These findings also have possible implications for the early detection of macular damage in diabetes. Because the macular region is rich in retinal ganglion cells, it could be suggested that diabetic damage of this central area might occur early in the disease process. In fact, animal models of DR have shown significant loss of macular ganglion cells [2428]. Moreover, the fractal dimension of the OPL and OS in MDR eyes was significantly smaller than similar measures in normal healthy eyes. Previous studies have shown that not only retinal pericytes and endothelial cells are susceptible to hyperglycemia, but neuroglial elements of the retinal are also involved in the retinal damage caused by diabetes. Interestingly, in the onedimensional fractal analysis performed using the same OCT data, the fractal dimension of the OPL in MDR eyes showed significantly higher values compared to healthy eyes [20]. The opposite conclusion might be due to Henle fiber layer artifacts that the thickness and the reflectance information might have been changed as the scanning is performed at oblique angles [29-30].

The ROC analysis was performed for the fractal dimension measures of each intraretinal layer. The AUROC values are shown in Table 3 by study groups.

Table 2 Distribution statistics of fractal dimension (mean $\pm \mathrm{SD}$ ) values by study group.

\begin{tabular}{cccc}
\hline Fractal Dimension & Controls & DM & MDR \\
\hline RNFL & $2.071 \pm 0.021$ & $2.078 \pm 0.015 \ddagger \S$ & $2.043 \pm 0.041 \ddagger \S$ \\
GCL+IPL & $2.219 \pm 0.020$ & $2.219 \pm 0.017 \ddagger$ & $2.202 \pm 0.031 \ddagger$ \\
INL & $2.104 \pm 0.028$ & $2.110 \pm 0.018 \ddagger \S$ & $2.089 \pm 0.030 \S$ \\
OPL & $2.155 \pm 0.026$ & $2.141 \pm 0.034 \ddagger \S$ & $2.111 \pm 0.024 \ddagger \S$ \\
ONL+IS & $2.138 \pm 0.038$ & $2.137 \pm 0.034 \ddagger \S$ & $2.107 \pm 0.037 \ddagger \S$ \\
OS & $2.075 \pm 0.015$ & $2.080 \pm 0.013 \ddagger \S$ & $2.053 \pm 0.016 \ddagger \S$ \\
RPE & $2.076 \pm 0.011$ & $2.077 \pm 0.008 \ddagger \S$ & $2.067 \pm 0.012 \ddagger \S$ \\
\hline
\end{tabular}

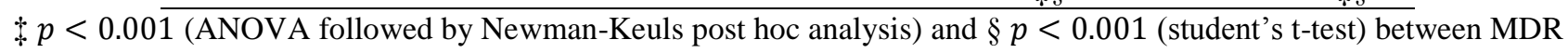
and healthy eyes (see MDR column) and between MDR and DM eyes (see DM column).

Table 3 AUROC values of best diagnostic parameters by study group.

\begin{tabular}{cccc}
\hline Intraretinal Layer & MDR vs. Controls & MDR vs. DM & DM vs. Controls \\
\hline RNFL & $0.744^{*}$ & $0.846^{* *}$ & 0.374 \\
GCL+IPL & 0.670 & 0.664 & 0.526 \\
INL & 0.652 & $0.711^{*}$ & 0.460 \\
OPL & $0.889^{* *}$ & $0.732^{*}$ & 0.598 \\
ONL+IS & $0.726^{*}$ & $0.712^{*}$ & 0.508 \\
OS & $0.844^{* *}$ & $0.906^{* *}$ & 0.392 \\
RPE & $0.706^{*}$ & $0.740^{*}$ & 0.496 \\
\hline
\end{tabular}

$0.7 \leq$ AUROC $\leq 0.8, * * 0.8 \leq$ AUROC. 
Detailed ROC analysis results for the fractal dimension that showed a significant difference between the groups are also shown in the Tables 4-5 and Figures 3-4. All AUROC values were greater than 0.5 when comparing MDR eyes to controls and DM eyes. The highest AUROC values estimated for the fractal dimension were observed for OPL and OS (0.889 and 0.844, respectively) when comparing MDR with normal healthy eyes. The cutoff point for the OPL was suggested to be 2.126 with a sensitivity and specificity of $83.3 \%$ and $74.4 \%$, respectively. The cutoff point for the OS was proposed to be 2.061 with a sensitivity and specificity of $79.7 \%$ and $69.8 \%$, respectively. The highest AUROC values estimated for the fractal dimension were observed for the RNFL and OS $(0.846$ and 0.906, respectively) when comparing MDR with DM eyes. The cutoff point for the RNFL was suggested to be 2.065 with a sensitivity and specificity of $81.6 \%$ and $72.1 \%$, respectively. The cutoff point for the OS was proposed to be 2.065 with a sensitivity and specificity of $86.8 \%$ and $76.7 \%$, respectively. The highest AUROC values were obtained for the OS when comparing MDR eyes with controls and DM eyes. This particular results might suggest that diabetes inflicts additional damage to the outer photoreceptor segment, which could be an early indication of visual function degeneration that could be used as an additional indicator to enable the early detection of diabetic retinal damage or disease progression [31-33].

There are some potential shortcomings of our study. Time domain OCT technology has some limitations compared to the more advanced OCT technology. Also, current OCT devices include different segmentation algorithms and methods for speckle noise removal. Therefore, data analysis is influenced by particular assumptions and technological specifications that are in place for each OCT device. Moreover, the box-counting method to calculate the fractal dimension has been reported as not the best technique to estimate fractal dimension when the image intensity surface is smooth [22,32]. Therefore, a modified differentiate box counting method should be considered to avoid the underestimation of fractal dimension. In the modified differentiate box-counting method, a random shift is added to the column in the vertical or horizontal direction when counting the number of the boxes for the 3D spatial surface. Though the random shifting in each column needs some additional computation time, the modified differentiate box counting method could generate the exact fractal dimension in smoothly textured images.

Table 4 Cutoff values derived from the ROC analyses between MDR group and Controls.

\begin{tabular}{cccccc}
\hline $\begin{array}{c}\text { Intraretinal } \\
\text { Layer }\end{array}$ & AUROC & $\begin{array}{c}\text { Asymptotic 95\% } \\
\text { CI } \\
\text { Lower-upper } \\
\text { bound }\end{array}$ & $\begin{array}{c}\text { Cutoff } \\
\text { Point }\end{array}$ & Sensitivity & Specificity \\
\hline RNFL & 0.744 & $0.651-0.836$ & 2.063 & $75.7 \%$ & $65.1 \%$ \\
GCL+IPL & 0.670 & $0.560-0.780$ & 2.214 & $70.3 \%$ & $60.5 \%$ \\
INL & 0.652 & $0.548-0.756$ & 2.098 & $66.2 \%$ & $55.8 \%$ \\
OPL & 0.889 & $0.831-0.947$ & 2.126 & $83.8 \%$ & $74.4 \%$ \\
ONL+IS & 0.726 & $0.630-0.821$ & 2.120 & $73.0 \%$ & $62.8 \%$ \\
OS & 0.844 & $0.769-0.919$ & 2.061 & $79.7 \%$ & $69.8 \%$ \\
RPE & 0.706 & $0.609-0.803$ & 2.071 & $67.6 \%$ & $58.1 \%$ \\
\hline
\end{tabular}

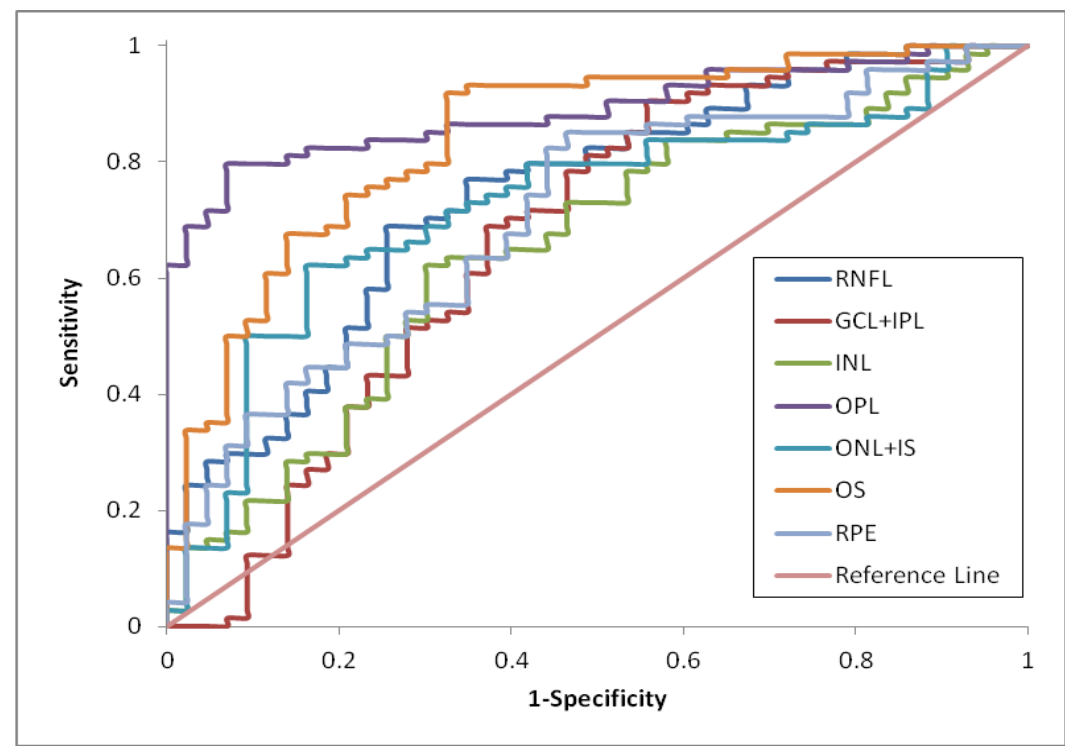

Fig. 3 Receiver operating characteristic (ROC) curves for the detection of diseased tissue using fractal dimension as the predictor variable when comparing MDR group to healthy group. 
Table 5 Cutoff values derived from the ROC analyses between MDR group and DM group.

\begin{tabular}{cccccc}
\hline $\begin{array}{c}\text { Intraretinal } \\
\text { Layer }\end{array}$ & AUROC & $\begin{array}{c}\text { Asymptotic } 95 \% \\
\text { CI } \\
\text { lower-upper } \\
\text { bound }\end{array}$ & $\begin{array}{c}\text { Cutoff } \\
\text { Point }\end{array}$ & Sensitivity & Specificity \\
\hline RNFL & 0.846 & $0.764-0.928$ & 2.065 & $81.6 \%$ & $72.1 \%$ \\
GCL+IPL & 0.664 & $0.544-0.784$ & 2.210 & $65.8 \%$ & $55.8 \%$ \\
INL & 0.711 & $0.598-0.824$ & 2.100 & $71.1 \%$ & $60.5 \%$ \\
OPL & 0.732 & $0.618-0.845$ & 2.119 & $63.2 \%$ & $53.5 \%$ \\
ONL+IS & 0.712 & $0.597-0.826$ & 2.111 & $65.8 \%$ & $55.8 \%$ \\
OS & 0.906 & $0.840-0.971$ & 2.065 & $86.8 \%$ & $76.7 \%$ \\
RPE & 0.740 & $0.631-0.848$ & 2.073 & $73.7 \%$ & $62.8 \%$ \\
\hline
\end{tabular}

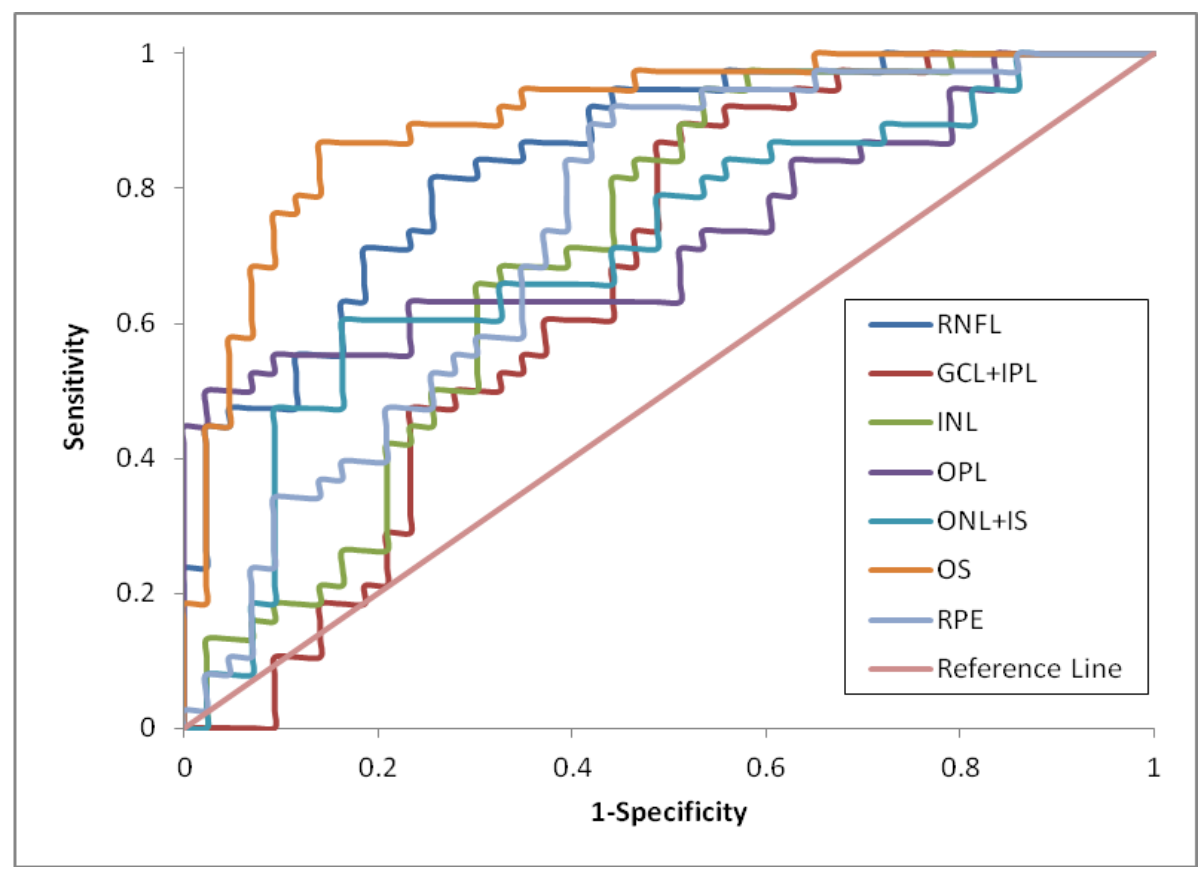

Fig. 4 Receiver operating characteristic (ROC) curves for the detection of diseased tissue using fractal dimension as the predictor variable when comparing MDR group to DM group.

Also, fractal analysis using the power spectrum technique, which is generalizable and potentially more precise, should be compared with the modified differentiate box counting method results to investigate which one produce better estimates in OCT imaging [34-35].

\section{Conclusion}

The early results presented have shown this methodology could have the potential to differentiate diabetic eyes with early retinopathy from normal healthy eyes. Particularly, this result is in agreement with previous reports showing a significant reduction of the fractal dimension during induced apoptosis throughout early apoptotic phases in breast cancer cells. This observation could be an early indication of visual function degeneration and could be used to improve early sign of diabetic retinal damage and disease progression. Future studies are needed to determine the accuracy, repeatability and full capability of this methodology with more OCT scans and advanced technology. A possibility of error exists due to the quantization nature of the approach, especially when the image intensity surface is smooth.

\section{Acknowledgement}

This study was supported in part by a Juvenile Diabetes Research Foundation Grant, the research grants NIH R01EY020607, NIH Center Grant P30 EY014801 and a grant from Research to Prevent Blindness (RPB), by a research fellowship of the Helen Keller Foundation for Research and Education, the Eötvös Scholarship of the Hungarian Scholarship Fund, by the Zsigmond Diabetes Fund of the Hungarian Academy of Sciences, by the Ministry of Education and Science of the Russian Federation, and by the research grant D2016009 from Ningbo University of Technology of China. 